\title{
Whole genome profiling of spontaneous and chemically induced mutations in Toxoplasma gondii
}

Andrew Farrell ${ }^{1 \dagger}$, Bradley I Coleman ${ }^{1 \dagger}$, Brian Benenati ${ }^{1}$, Kevin M Brown ${ }^{2,3}$, Ira J Blader ${ }^{2,4}$, Gabor T Marth and Marc-Jan Gubbels ${ }^{1 *}$

\begin{abstract}
Background: Next generation sequencing is helping to overcome limitations in organisms less accessible to classical or reverse genetic methods by facilitating whole genome mutational analysis studies. One traditionally intractable group, the Apicomplexa, contains several important pathogenic protozoan parasites, including the Plasmodium species that cause malaria.

Here we apply whole genome analysis methods to the relatively accessible model apicomplexan, Toxoplasma gondii, to optimize forward genetic methods for chemical mutagenesis using $\mathrm{N}$-ethyl-N-nitrosourea (ENU) and ethylmethane sulfonate (EMS) at varying dosages.
\end{abstract}

Results: By comparing three different lab-strains we show that spontaneously generated mutations reflect genome composition, without nucleotide bias. However, the single nucleotide variations (SNVs) are not distributed randomly over the genome; most of these mutations reside either in non-coding sequence or are silent with respect to protein coding. This is in contrast to the random genomic distribution of mutations induced by chemical mutagenesis. Additionally, we report a genome wide transition vs transversion ratio (ti/tv) of 0.91 for spontaneous mutations in Toxoplasma, with a slightly higher rate of 1.20 and 1.06 for variants induced by ENU and EMS respectively. We also show that in the Toxoplasma system, surprisingly, both ENU and EMS have a proclivity for inducing mutations at A/T base pairs (78.6\% and $69.6 \%$, respectively).

Conclusions: The number of SNVs between related laboratory strains is relatively low and managed by purifying selection away from changes to amino acid sequence. From an experimental mutagenesis point of view, both ENU (24.7\%) and EMS (29.1\%) are more likely to generate variation within exons than would naturally accumulate over time in culture (19.1\%), demonstrating the utility of these approaches for yielding proportionally greater changes to the amino acid sequence. These results will not only direct the methods of future chemical mutagenesis in Toxoplasma, but also aid in designing forward genetic approaches in less accessible pathogenic protozoa as well.

Keywords: Whole genome sequencing, Chemical mutagenesis, In vitro adaptation, SNV calling, Apicomplexa

\footnotetext{
* Correspondence: gubbelsj@bc.edu

'Equal contributors

'Department of Biology, Boston College, Higgins Hall 355, 140

Commonwealth Avenue, Chestnut Hill, MA 02467, USA

Full list of author information is available at the end of the article
} 


\section{Background}

The Apicomplexa comprise important human pathogens such as the malaria-causing Plasmodium spp. [1] and Toxoplasma gondii, which can cause life-threatening opportunistic disease and birth defects [2]. Due to the complex life cycle of most Apicomplexa, the experimental accessibility of these parasites has been limited. However, Toxoplasma is a comparatively accessible model for other apicomplexan parasites [3]. The development of Toxoplasma as a forward genetic system was pioneered in the 1970s by Elmer Pfefferkorn, who took advantage of the ability to culture the asexual tachyzoite life cycle stage indefinitely in vitro and the parasite's short generation time of $\sim 7$ hrs. Using mutagenized parasites Pfefferkorn started to dissect the nucleotide salvage and synthesis pathways $([4,5]$ reviewed in [6]). In the years since Pfefferkorn's original work, chemical mutagenesis and forward genetic analyses have been successfully applied to various unique aspects of Toxoplasma biology, including invasion and egress from the host cell and internal budding, the parasite's distinct mode of cell division [6-14].

Full exploitation of the power of Toxoplasma forward genetics will require a better understanding of the mutagenic profiles associated with specific mutagenesis protocols. Pfefferkorn initially used N-ethyl-N-nitrosourea (ENU) on actively growing intracellular parasites. Efficient mutagenesis was also obtained through nitrosoguanidine treatment of extracellular parasites and ethylmethane sulfonate (EMS) treatment of intracellular parasites [15]. ENU and EMS are both widely used chemical mutagens: EMS is favored in genetic studies in plants [16], fruit flies [17] and C. elegans [18], whereas ENU is preferentially used in mice [19] and zebrafish [20]. The choice of mutagen is dependent upon the organism's DNA composition, DNA repair pathway efficiencies, and the chemical's mutagenic signature. As a rule of thumb, EMS preferentially results in $\mathrm{G} / \mathrm{C}$ base pair mutations whereas ENU has a bias toward A/T base pairs [21-23]. We previously confirmed the A/T proclivity of ENU in a single Toxoplasma mutant [7], but the EMS mutagenic signature is currently unknown.

Whole genome sequencing (WGS) has been used in model organisms such as Caenorhabditis elegans [18] and Saccharomyces cerevisiae $[24,25]$ to identify the numbers of mutations per genome, the ratio of silent vs. non-silent mutations, the chance of generating mono-allelic traits and other genome-wide analyses. By cataloging the mutations in a single genome associated with a specific phenotype, these approaches also allow mapping of phenotypic traits to genomic loci. This has been best explored and defined in C. elegans [18,24,26-29], but there are examples from zebrafish [30] and S. cerevisiae [25,31] as well. WGS and analysis of the genome-wide distribution of mutations are new tools whose power has been recognized in genetic model organisms [32]. In developing genetic systems for non-model organisms, however, this power has not been fully exploited.

In Toxoplasma, the combination of forward genetics and WGS has extended Pfefferkorn's pioneering chemical mutagenesis studies to map drug resistance genes $[33,34]$ and biological phenotypes in invasion and egress $[7,13]$. To further the development of the Toxoplasma genetic system, we previously initiated an optimization of forward mutagenic protocols [35]. Here we further exploit the power of WGS to expand on these efforts by defining the mutagenic profiles of ENU and EMS at varying dosages. Through the analysis of 1208 single nucleotide variations (SNVs) spontaneously generated in Toxoplasma under lab conditions, we also show that these mutations reflect genome nucleotide composition, without any bias. Furthermore, we show that both ENU (369 SNVs) and EMS (158 SNVs) have a proclivity for inducing mutations at $\mathrm{A} / \mathrm{T}$ base pairs while also generating greater proportions of protein code changing SNVs than those generated spontaneously during in vitro culture. Finally, we show there are no apparent hot- or cold-spots within the genome for variations generated via either in vitro culture or chemical mutagenesis. We use these insights to design an optimized chemical mutagenesis protocol for forward genetic experiments in Toxoplasma and potentially other Apicomplexa.

\section{Methods \\ Parasites}

Parasites were maintained by in vitro passage in human foreskin fibroblasts (HFF cells) [36]. An overview of the genealogy of these strains is given in Figure 1. All the strains are derived from the Type I RH Toxoplasma strain isolated from a 1939 case of toxoplasmic encephalitis [37] and subsequently cloned and adapted for in vitro culture in the 1970s [5]. The RH-HXGPRT knock-out strain (RH- $\triangle \mathrm{HXGPRT)}$ was generated by homologous recombination and 6-thioxanthine selection in the 1990s [38]. The RH- $\triangle$ HXGPRT strain made its way from the Roos lab to the Boothroyd and Striepen labs, and from there to the Blader and Gubbels labs, respectively. For the purpose of this paper these sibling strains are referred to as the 'B-RH' and 'G-RH' strains. The transgenic $2 \mathrm{~F}$ line stably expresses LacZ ( $\beta$-galactosidase), which was selected for stable, random genomic integration by phleomycin through the BLE selectable marker [39]. The $2 \mathrm{~F}$ line was recloned around 2000 by Vern Carruthers to make 2F-1 (also referred to as 2F1 [40]). Subsequently, chloramphenicol selection for the CAT selectable marker was applied to stably integrate a tandem YFP expressing plasmid resulting in 2F-1-YFP2 [41]. The number of passages (each representing $\sim 8$ generations) along the journeys of these strains is unknown, but is likely in the range of 100-1000s. 


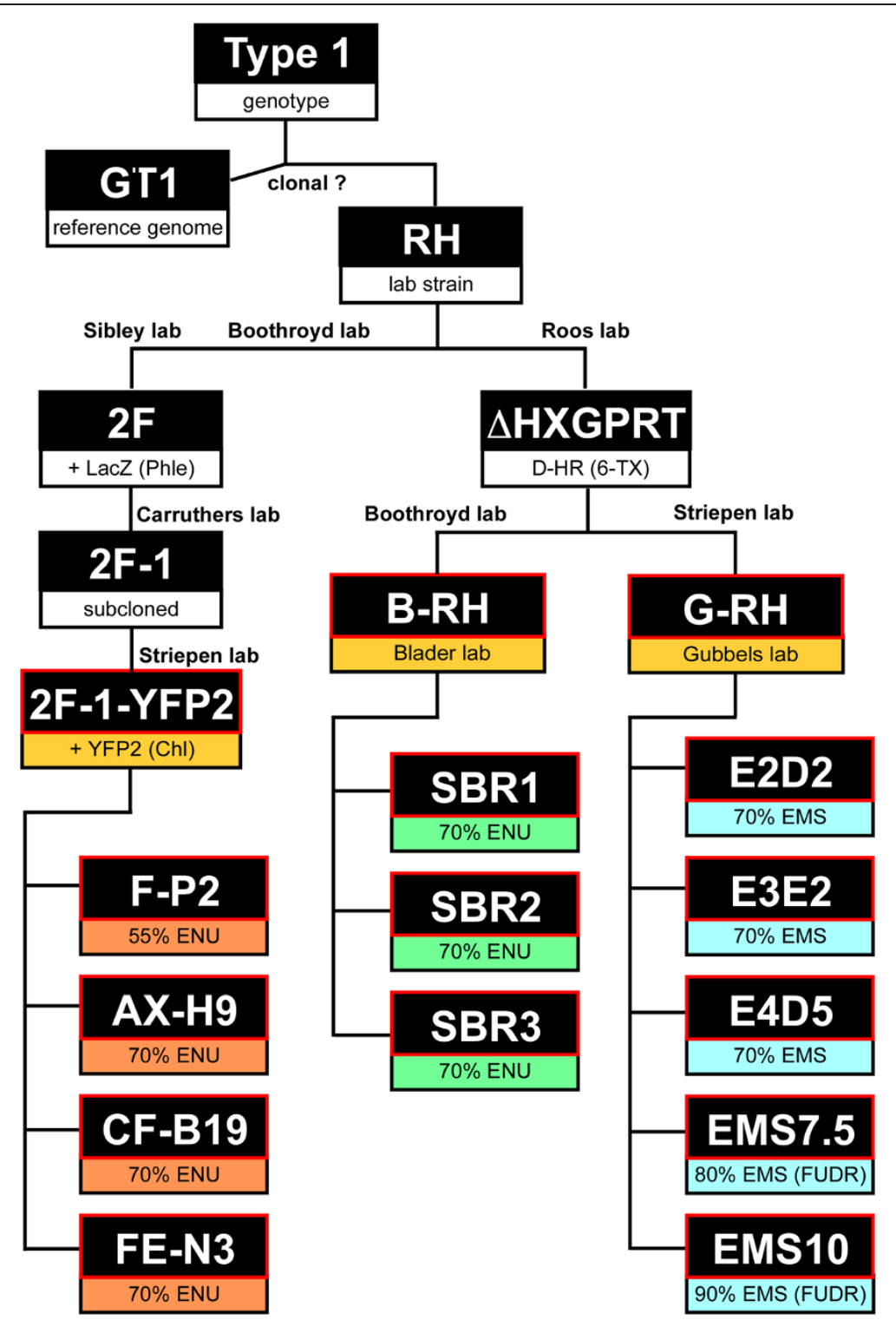

Figure 1 Lineage overview of the parasite strains used in this study. All are of the Type I genotype, with GT1 the sequenced reference genome available on ToxoDB.org [48]. The GT1 and RH strains are most likely descendants from the same clone [49]. All sequenced strains and mutants are outlined by red boxes; all are derived from the RH strain. Drug selections to generate transgenic lines are given between brackets in the lower box of each line. Mutagen dosage is expressed as the percentages of parasites being killed by mutagen treatment (measured by plaque assays). Phle: phleomycin; D-HR: double homologous recombination; 6-TX: 6-thioxanthine; Chl: chloramphenicol; FUDR: 5-fluoro-2'-deoxyuridine; HXGPRT: hypoxanthine-xanthine-guanine-phosphoribosyl transferase.

\section{Mutagenesis and phenotype screening}

Chemical mutagenesis was performed essentially as described in [35]. Briefly, a T25 flask of confluent HFFs was inoculated with $1 \mathrm{ml}$ of freshly lysed $T$. gondii, which were allowed to invade and replicate in Ed1 media for $18-25 \mathrm{hrs}$ at $37^{\circ} \mathrm{C}$. Ed1 was replaced with $10 \mathrm{ml}$ of $0.1 \mathrm{X}$ Ed1 (diluted in DMEM) and the flasks returned to $37^{\circ} \mathrm{C}$ for 10 minutes. Intracellular parasites were treated with the mutagen of choice and the appropriate vehicle controls for $4 \mathrm{hrs}$ at $37^{\circ} \mathrm{C}$. After exposure to mutagen, parasites were recovered by washing the monolayer $3 \mathrm{X}$ with cold PBS, scraping and lysing the host cells via passage through a $26.5 \mathrm{G}$ needle and filtration through a $3.0 \mu \mathrm{m}$ polycarbonate filter. Parasites were recovered by centrifugation at $1000 \times \mathrm{g}$ for $10 \mathrm{~min}$ and returned to a new confluent T25 flask. The mutagenized population was allowed to lyse the monolayer naturally, typically in 5-7 days. Percent killing was determined by serial dilution of 10,000 mutagenized and vehicle treated parasites into 6 well plates confluent with HFF cells. After 1 week of undisturbed growth, wells were stained for the presence of plaques. Percentages were calculated as a ratio 
of plaque number in treated parasites vs. the vehicle control. Following phenotypic selection of mutants, individual parasite clones were isolated through serial dilution in 384 well plates of confluent HFFs. After 7 days, wells containing only single plaques, as seen through the microscope, were considered monoclonal populations.

ENU mutants were generated in different genetic backgrounds at concentrations of ENU in the range of 3 $7 \mathrm{mM}$ (Figure 1; because ENU is unstable, stocks should be titrated relative to percent killing as described above [35]). Mutants F-P2, AX-H9, CF-B19, and FE-N3 were generated by Gubbels and screened for a temperature sensitive growth defect (permissive and restrictive temperatures were $35^{\circ} \mathrm{C}$ and $40^{\circ} \mathrm{C}$, respectively) [8]; mutants SBR1-3 were generated by the Blader lab and screened for resistance against the kinase inhibitor SB505124 [33]. Mutant F-P2 was generated using a 55\% killing dose; for all others a dose inducing 70\% killing was used. All mutants were generated by independent mutagenesis experiments and therefore represent unique SNV pools. For this study we resequenced mutant F-P2 with longer reads. We differentiate the "old" data from the "new" data as o-F-P2 [7] and n-F-P2. The two DNA isolations were performed two passages apart, which corresponds to approximately 16 generations.

EMS mutants were obtained at mutagen dosages ranging from 3-10 $\mathrm{mM}$ in the G-RH genetic background. Again, as above, the mutagen was titrated via percent killing. Mutants E2D2, E3E2, and E4D5 were generated at 70\% killing. E2D2 and E4D5 were screened for resistance against DTT induced egress [42] whereas E3E2 was screened for resistance against the invasion enhancing compound $2[42,43]$. Mutants EMS7.5 and EMS10 were generated using 7.5 and $10 \mathrm{mM}$ EMS, inducing $80 \%$ and $90 \%$ killing, respectively and screened for resistance against $20 \mu \mathrm{M}$ FUDR [15]. All EMS mutants were also generated by independent mutagenesis experiments.

\section{Whole genome sequencing}

Parasites were collected $24 \mathrm{hrs}$ after complete lysis of the HFF cell monolayer without further scraping, passed three times through a $26.5 \mathrm{G}$ needle, and filtered through a $3 \mu \mathrm{m}$ filter membrane. Harvesting parasites $24 \mathrm{hrs}$ post host cell lysis, while taking care not to disturb the HFF monolayer, largely eliminates the $50 \%$ host DNA background we previously observed [7]. Genomic DNA was prepared with the Qiamp DNA mini kit (Qiagen; Valencia, CA) using the manufacturer's protocol for cultured cells. Illumina sequencing of strains 2F-1-YFP2 and o-F-P2 was performed at the Broad Institute, Cambridge, MA as described in [7] and all others at the Arthritis \& Clinical Immunology Department of the Oklahoma Medical Research Foundation, Oklahoma City, OK as described in [33].

\section{SNV calling}

Methods used here are essentially the same as have been previously described [7]. FASTQ sequence traces were aligned to a FASTA reference containing both the Toxoplasma gondii GT1 genomic reference v7.0 and the human genome reference build 37. Alignments were performed with MOSAIK using -mmp .15, -mhp 100 -act 35 -hs 15. Variable descriptions and procedures are described in the documentation V1.0 (available at http://bioinformatics. bc.edu/marthlab/wiki/index.php/Software) [44]. Variations were called with FreeBayes using standard parameters as described in the documentation, software version 0.9.9 $[45,46]$. The resulting SNV calls for the respective samples were compared to the SNV calls for its respective parent sample to identify both variations that were shared between the two samples and those that were unique to the mutant. Calls were filtered to remove SNVs with coverage less than $5 \mathrm{X}$ in either sample, a $\mathrm{P}$ value less than 0.8 , and less than $75 \%$ allele balance in either samples at that position.

To compare the three parent samples, 2F-1-YFP2, $\mathrm{B}-\mathrm{RH}$, and $\mathrm{G}-\mathrm{RH}$, each parent was compared to each of the other two parents separately as described above, creating a total of 3 datasets: n-F-P2 vs. B-RH, n-F-P2 vs. $\mathrm{G}-\mathrm{RH}$, and $\mathrm{B}-\mathrm{RH}$ vs. G-RH (note that n-F-P2 sequence data was used instead of 2F-1-YFP2 since its coverage is greater and the error rate and human contamination are much lower; F-P2 specific mutations [7] were manually removed after analysis). In each of the samples shared and unique SNVs from each comparison were pooled together to create a complete list of variants for each sample. To create a high confidence unified set for three samples, the rejected positions for all three comparisons were pooled and SNV calls that intersected with any rejected position were removed from the variant lists.

\section{Other computational methods}

Circose plots were generated using Circos as described in [47]. Toxoplasma gene densities were calculated using GFF data for GT1 v7.0 available from toxodb.org [48]. Percentages were calculated by counting the number of bases within regions annotated as "gene" in a $100 \mathrm{~kb}$ window.

\section{SNV validation}

PCR primers were designed to amplify $250 \mathrm{bp}$ up- and down-stream of a select number of called mutations (further details and primer sequences provided in Additional file 1: Table S1). Primers were designed using an automated algorithm that required primers to fit the following description: total product size of $500 \pm 200$ base pairs, primer GC content between $40 \%$ and $50 \%$, the 3' most base must be either a $\mathrm{G}$ or $\mathrm{C}$, of the last five 3'-basepairs three must be either $\mathrm{A}$ or $\mathrm{T}$, and the primer must be unique within the Toxoplasma genome. Primers are 
initially designed as 20 bases, if a primer could not be found that satisfied the above criteria at that length the calculations were run again with 19 bases, then 21 bases and so on. Furthermore, the sense primers were designed with a 5' extension composed of the M13 universal primer. PCR products were amplified from parent and mutant genomic DNA and analyzed by agarose gel electrophoresis. PCR bands were extracted from gel and sequenced using the M13 universal primer.

\section{Results}

\section{Sequencing results}

Illumina paired-end whole genome sequence reads were obtained for all strains outlined by red boxes in Figure 1. Collected reads were aligned to the Toxoplasma GT1 reference strain genome assembly [48]. The GT1 strain was isolated from goat skeletal muscle and is a Type I genotype strain closely related to the $\mathrm{RH}$ strain, for which no fully sequenced reference currently exists [49]. Sequences were obtained on different machines in different facilities, producing different read lengths and qualities (Figure 2). Regardless of differences in read length, genomic coverage was very high with more than $99.5 \%$ of the reference genome covered at a read depth of 10 or greater. Average genome coverage ranged from a depth of 29 for SBR1 to 119 for n-F-P2. From a practical perspective it is important to cover as much of the genome as possible when looking for a causative mutation. Our alignment covers $>99.5 \%$ of the sequenced GT1 reference, but because gaps remain in the completed sequence, parts of the genome might be absent from our analyses. To assess how much of the genome may be missing from the reference we identified the proportions of our total reads that aligned to the 14 chromosomal contigs, to the 365 unplaced contigs or were unaligned. Less than $1 \%$ of the reads aligned to the 365 unaligned contigs, implying that these contigs do not account for more than $1 \%$ of the true genome. Between $2 \%$ and $10 \%$ of the sequence reads could neither be aligned to the GT1 reference, nor to the human (host cell) genome. A significant percentage of unaligned reads is common and expected in Illumina sequence; these reads likely represent simple sequencing errors, not significantly large missing parts of the $\mathrm{RH}$ genome. Previous studies report alignment percentages from as low as $70 \%$ up to $94 \%[24,27,50-52]$. We therefore estimate that the 14 complete contigs in the Toxoplasma reference likely comprise as much as $99 \%$ of the true genome. Whether the remaining $1 \%$ represents a true chromosomal discrepancy between GT1 and RH is not further pursued here.

\section{SNV calling}

We called SNVs in all samples using FREEBAYES. Our previous work has shown that numerous areas in the GT1 reference appear to be either duplicated or assembled incorrectly when compared to our RH strains. This produces incorrect read alignments and leads to a high rate of spurious variant calls. As we previously published, to filter out potentially spurious SNV calls in these regions, mutant samples were compared to the relevant parent line to remove areas that had poor alignments in either sample [7]. In that work, after filtering there were 997 polymorphisms shared between the o-F-P2 and 2F-1-YFP2 parent, and 33 candidate mutations unique to F-P2. Fifteen of the SNVs removed by filtering were randomly selected and all were experimentally confirmed as false positives. 31 of 33 mutations called as unique in mutant F-P2 (using the o-F-P2 data) were validated by PCR amplification and Sanger sequencing [7].

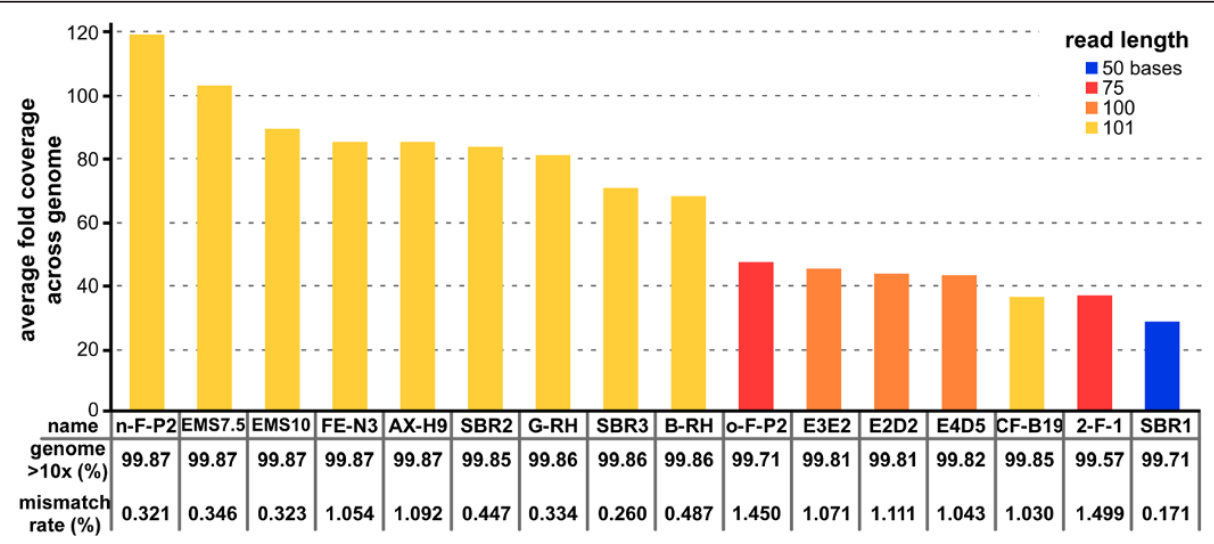

Figure 2 Genomic coverage and quality of sequencing reads. Average fold genomic coverage for the various strains is plotted as indicated. Bar color reflects the Illumina read length used as indicated in the legend. In the Table in the lower half the percentage of the genome covered at least 10-fold is shown as percentage of the complete GT1 reference genome (in all cases over 99.5\%). Read quality is reported as the mismatch rate between reads and the reference genome. $\mathrm{n}-\mathrm{F}-\mathrm{P} 2[\mathrm{n}=\mathrm{new}]$ is a re-sequenced sample of the F-P2 mutant with longer read length [7], named o-F-P2 [o = old]. 
Of the two remaining false positive calls, one was eliminated by using the n-F-P2 sequence reads produced for this study, which are of lower error rate, deeper coverage and longer read length. For data generated in this study, we validated a select number of SNV calls in EMS mutants E2D2, E3E2, and E4D5 (Additional file 1: Table S1). The data for 30 variations identified no false positive calls. Hence, the false positive rate based on these sets of data is 1 out of 63 SNVs $(33+30$ total tested), which is $1.58 \%$. Although the false positive rate is low, and may represent an overestimation given that no false positives were confirmed in the new dataset, it should be considered in the interpretation of the presented data.

To identify differences between the three parental strains each sample was compared against the two other parent samples. For each strain, the mutations (relative to the reference) that were found only in that parent were combined with those mutations shared amongst the parental strains to create a complete high quality set of SNVs for that sample. To ensure equal confidence in the data from all three samples, SNVs occurring at positions rejected in any of the samples were removed from all three mutants. This is a more conservative set of calls than the paired set, as any reduction in coverage in one sample will affect calls in all three, but ensures an unbiased comparison between the three samples. The parent sample 2F-1-YFP2 has by far the highest mismatch rate and second lowest coverage of all of samples. To improve the quality of the parent comparison, the well characterized sample n-F-P2 [7], of which 2F-1-YFP2 is the parental strain, was substituted and the 31 confirmed SNVs caused by ENU mutagenesis were removed. SNV calls and further analyses for all strains and mutants are available in Additional file 2: Table S2.

\section{Natural variations in non-mutagenized parent laboratory strains}

Reads of the three $\mathrm{RH}$-derived parent strains are analyzed here: 2F-1-YFP2, B-RH and G-RH (Figure 1). Compared to GT1, 984 mutations are shared among all three (50 of these are in the apicoplast genome). In this dataset, the rate of transitions (i.e. mutations of $\mathrm{T} / \mathrm{A}$ to $\mathrm{C} / \mathrm{G}$ and from $\mathrm{G} / \mathrm{C}$ to $\mathrm{A} / \mathrm{T}$ ) to transversions (T/A to $\mathrm{G} / \mathrm{C}, \mathrm{T} / \mathrm{A}$ to $\mathrm{A} / \mathrm{T}$, $\mathrm{G} / \mathrm{C}$ to $\mathrm{T} / \mathrm{A}$ or $\mathrm{G} / \mathrm{C}$ to $\mathrm{C} / \mathrm{G}$ ) was 0.92 (Figure 3 ). The ti/tv rate is unique for each species and possibly reflects the nature of DNA repair activity in the species [53]. Because of the wobble base pairing of codon and anti-codon in the ribosome, transitions result in more radical amino acid changes than transversions [54]. These variations could be the result of differences between the primary RH and GT1 isolates, but are more likely to have accumulated during the extensive lab maintenance of the $\mathrm{RH}$ strain before these three parental lines diverged. In addition to the single nucleotide mutations shared among all three lines in our dataset, the sibling RH- $\triangle \mathrm{HXGPRT}$ strains share an additional 85 SNVs not found in 2F-1-YFP2. Beyond this, B-RH contains 66 completely unique SNVs while G-RH has 19. 2F-1-YFP differs from the GT1 reference by 54 unique SNVs. When these numbers are compared to the number of SNVs shared among all three strains (984), this suggests that of all SNVs at least $54 / 1038 \times 100=5.2 \%$ for 2F-1-YFP2, $(85+66) / 1135 \times 100=13.3 \%$ for B-RH, and $(85+19) / 1088 \times 100=9.6 \%$ for G-RH have accumulated during in vitro growth under lab conditions.

We also assessed the impact of the mutations on protein coding (Figure 3B). We observed that $14.3 \%$ of the shared variations (141 out of 984) cause a change in amino acid sequence; either a non-synonymous mutation or the generation of a non-sense codon. $47 \mathrm{SNVs}$ cause synonymous mutations. Thus, $19.1 \%$ of SNVs caused a mutation within protein coding sequence $(141+47$ out of 984 ; Figure $3 B)$. The differences between the two $\mathrm{RH}$ strains have accumulated during laboratory maintenance, as they originate from the same clonal line [38]. The 85 variations shared between the B-RH and G-RH strains not found in 2F-1YFP2 suggest that either the parent $\mathrm{RH}$ strain used to generate the HXGPRT deletion diverged from the RH strain used to generate the $2 \mathrm{~F}$ strain, or that the $\mathrm{RH}$ $\triangle$ HXGPRT strain accumulated a significant number of mutations before the strain was shared between the Roos and Boothroyd labs.

\section{ENU mutagenic profile}

A single ENU mutant was created at a dose resulting in 55\% killing (mutant F-P2) and six mutants were derived from a $70 \%$ killing dose (all other ENU mutants) (Figure 1). For mutants generated at 70\% killing, the number of mutations varies from 23 (SBR2) to 70 (SBR1), with an average of 56.2 (Figure 4A). SBR2 is a likely outlier in the $70 \%$ set, lying even below the $55 \%$ killing data point ( $32 \mathrm{SNVs}$ ). Omitting SBR2 results in an average of 62.8 SNVs at 70\% killing. In either case, the variability at one of only two doses prevents a robust correlation between mutagen dosage and number of mutations.

When we analyze the bases targeted by ENU we find that $78.6 \%$ of mutations are found at $A$ or $T$ (Figure $4 B$ ). This is quite different from the random nucleotide distribution observed during in vitro culture where $44.5 \%$ of the SNVs are at A or T (Figure 3D), close to the natural incidence in the genome (45\% $\mathrm{A}$ or $\mathrm{T})$ [55]. ENU mutagenesis generated a ti/tv ratio of 1.2 , slightly higher than observed in the spontaneous mutations (Figure 4B).

\section{EMS mutagenic profile}

We generated EMS mutants at dosages inducing 70\%, $80 \%$ and $90 \%$ killing. In order to inform future genetic studies, we used these data to determine the relationship between the dose of EMS and the number and type of 


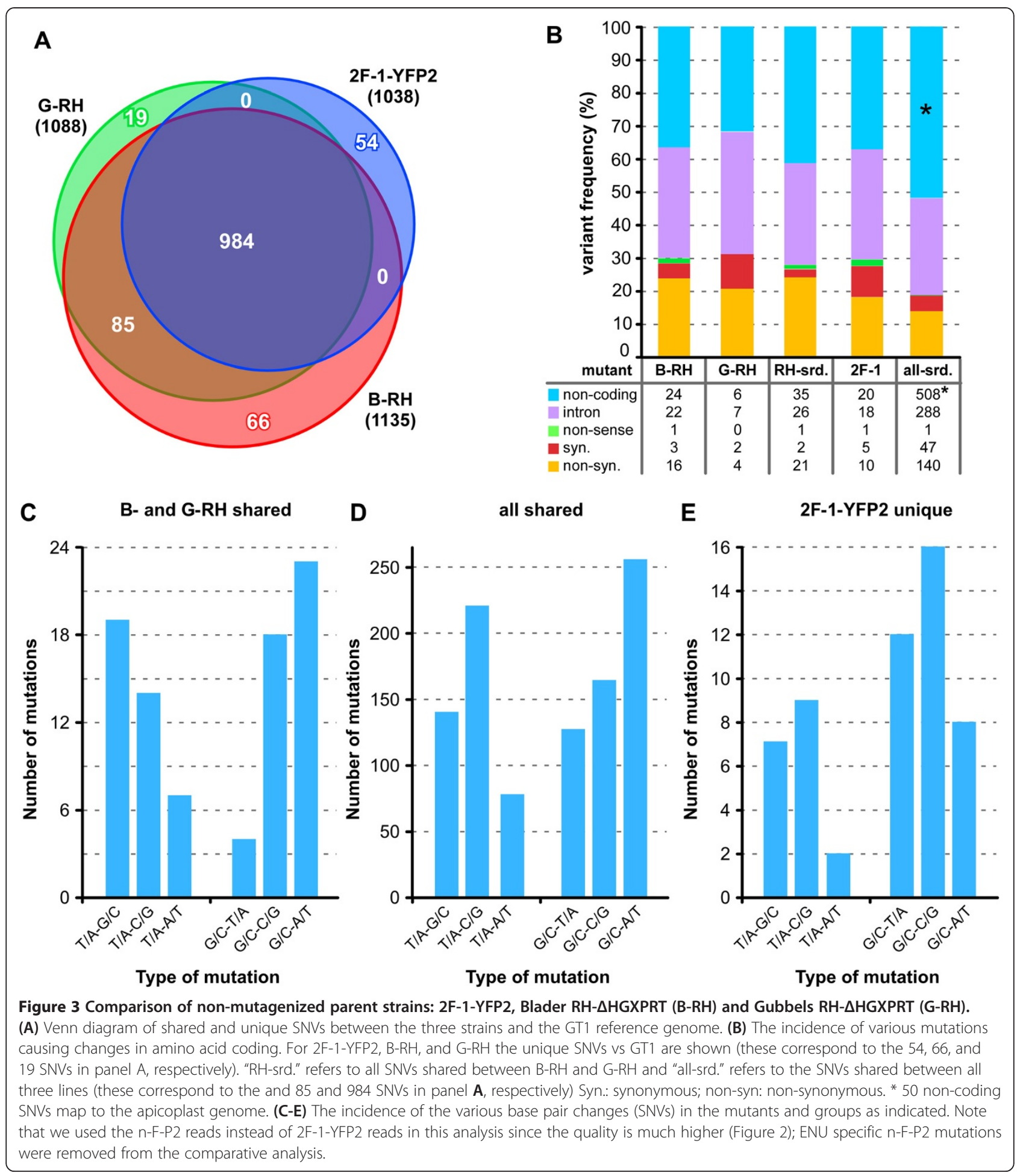

resulting mutations. At 70\% killing, the number of SNVs was very consistent, with 18,15 and 18 mutations identified in E2D2, E3E2 and E4D5, respectively. At 80\% killing (mutant EMS7.5) this number increased to $37 \mathrm{SNVs}$ and further increased to $70 \mathrm{SNVs}$ at $90 \%$ killing (mutant EMS10) (Figure 5A). These data show that the number of mutations induced by EMS approximately doubles as killing increases by $10 \%$.

Across all EMS mutants $23.4 \%$ of the SNVs represented non-synonymous mutations (Figure 5A), which was slightly higher than the $19.8 \%$ observed for ENU mutagenesis (Figure 4A). Thus, both chemical mutagens 


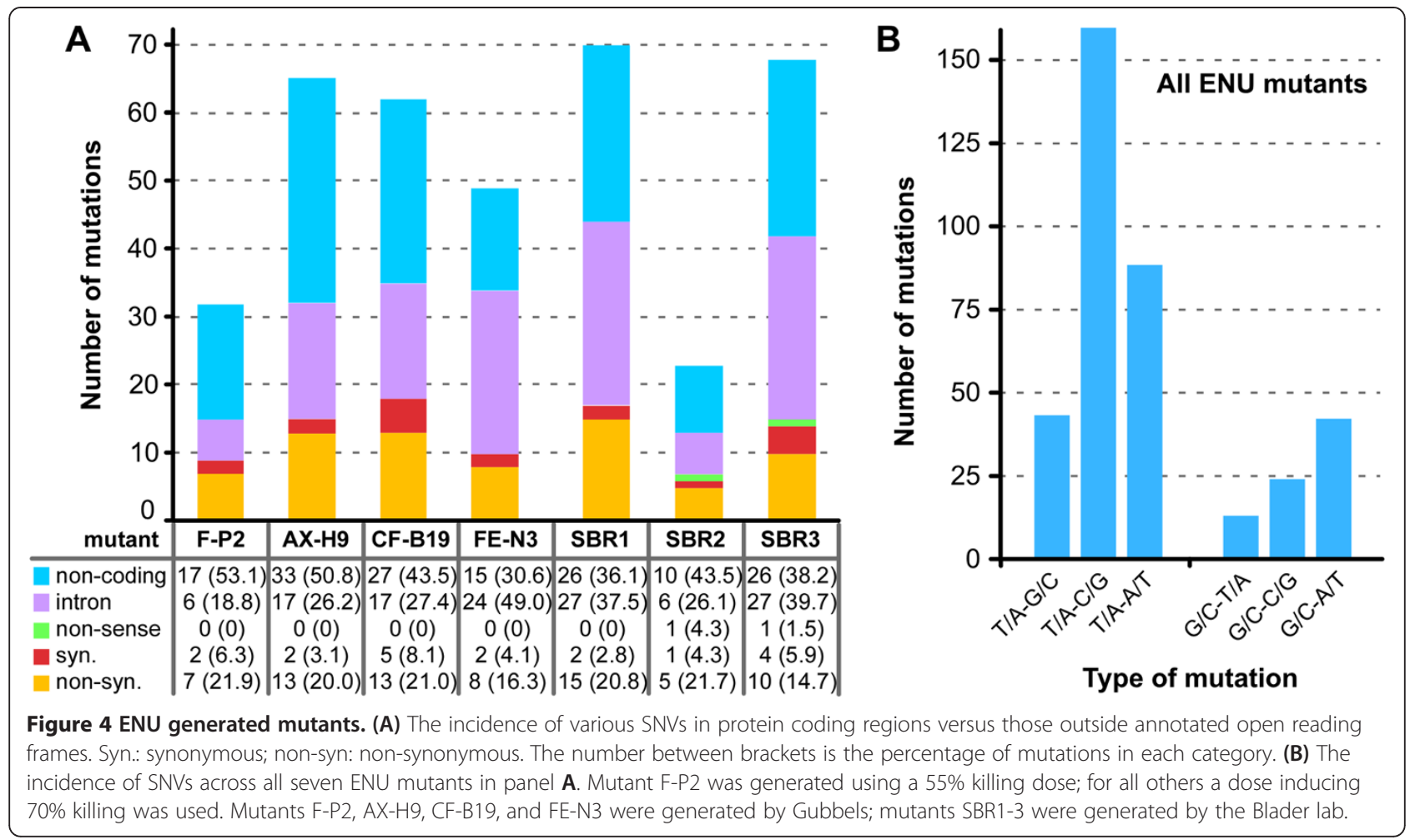

led to increased levels of non-synonymous mutations relative to the $14.3 \%$ observed during long term in vitro growth (Figure 3B). EMS mutants showed a ti/tv rate of 1.06. This was slightly lower than that of ENU but still higher than the rate observed for spontaneous mutations. Importantly, although the ti/tv rate is lower, the number of non-synonymous mutations is actually higher for EMS compared to ENU.

Following mutagenesis, the $80 \%$ and $90 \%$ killing samples were selected via induction of resistance to FUDR. This is a nucleotide analogue of deoxyuridine, and could therefore potentially have a mutagenic effect. Uracil
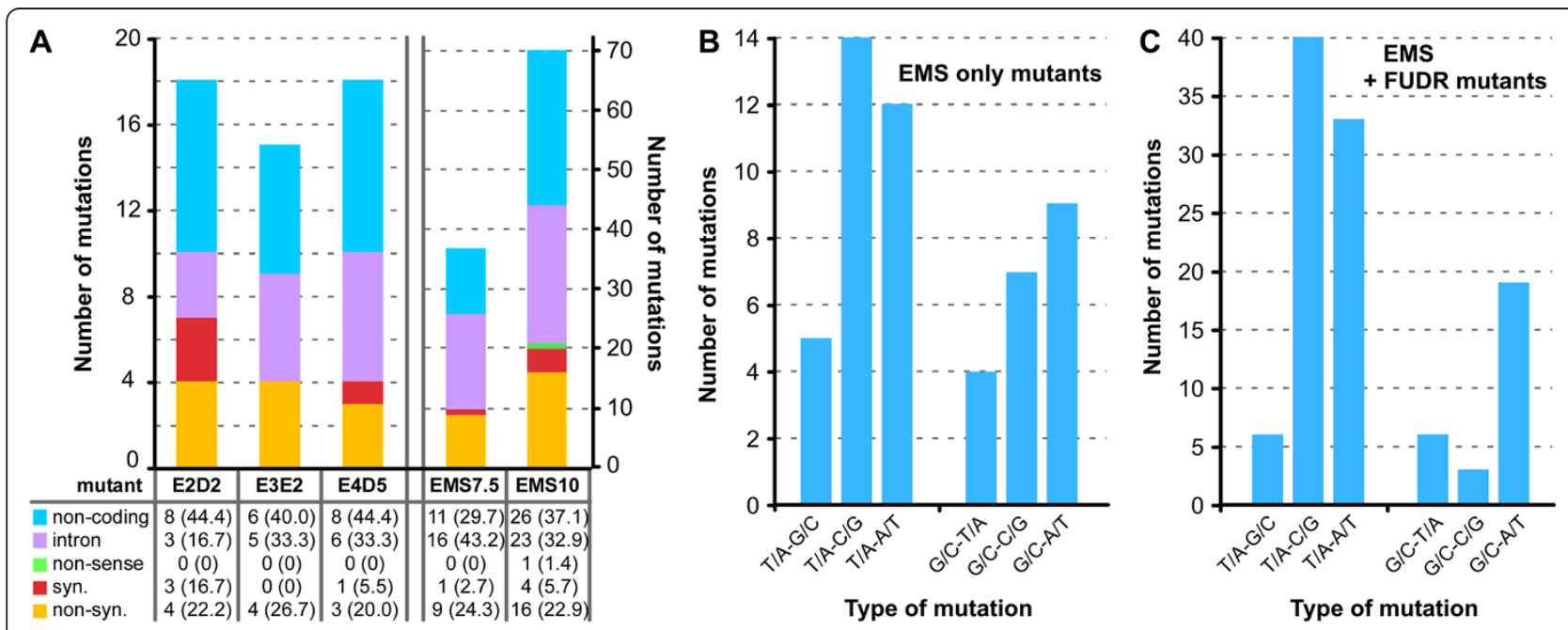

Figure 5 EMS generated mutants. (A) The incidence of various SNVs in protein coding regions versus those outside annotated open reading frames. Syn.: synonymous; non-syn: non-synonymous. The number between brackets is the percentage of mutations in each category. (B) The incidence of SNVs across the EMS mutants screened for resistance to pharmacologically induced egress using DTT (E2D2 and E4D5) or invasion enhancing compound 2 (E3E2) [42,43]. These three mutants were generated using an EMS dosage inducing $70 \%$ killing. (C) The incidence of SNVs across the EMS mutants screened for resistance against 20 MM FUDR [15]. EMS7.5 and EMS10 were generated using 7.5 and 10 mM EMS, inducing $80 \%$ and $90 \%$ killing, respectively. 
phosphoribosyl transferase (UPRT) converts FUDR to fluorodeoxyuridylic acid, a suicide inhibitor of thymidilate synthase [6]. It is therefore possible that usage of FUDR or its metabolic derivatives could result in DNA mutations that skew the mutagenic profile. As such, we considered the base preferences for the EMS mutants separately for those mutants generated in the absence and presence of FUDR selection (Figure $5 \mathrm{~B}$ and $5 \mathrm{C}$, respectively). The percentage of mutations found at A/T bases was $60.8 \%$ for mutants generated with EMS alone (51 total SNVs) and $73.8 \%$ for those additionally selected with FUDR (107 SNVs). The ti/tv ratio in the EMS alone mutants was 0.8 , while for the FUDR selected strains $\mathrm{ti} / \mathrm{tv}$ was 1.2. It appears that selection with FUDR may have the potential to influence the EMS mutagenic profile. However, the subtlety of this effect, combined with the small sample size means that any effects of FUDR on the mutagenic profile of our SNV pool are minor. Therefore, the FUDR treated and untreated EMS mutagenized samples have been considered together in our analyses, as the effects of EMS are certain to be the more significant source of mutations than the selection with FUDR (an increase in EMS dosage by 10\% in killing rate doubles the number of SNVs regardless of whether FUDR is present).

\section{Characterization of FUDR resistant parasites}

Mutants EMS7.5 and EMS10 represent FUDR resistant clones identified by screening against $20 \mu \mathrm{M}$ FUDR. To more specifically evaluate the FUDR resistant phenotypes we determined the IC50 value of the EMS mutants and compared them with the parent $\mathrm{RH}$ strain and parasites wherein the UPRT locus was genetically deleted $(\triangle \mathrm{UPRT})$. It has been shown that FUDR resistance can be mediated by mutations in the UPRT gene and that FUDR resistant mutants have no detectable UPRT activity [56]. UPRT functions in the pyrimidine salvage pathway, which is not essential in Toxoplasma because it can synthesize its own pyrimidines [57]. The FUDR IC50 for wild type parasites is less than $0.3 \mu \mathrm{M}$, and for $\triangle \mathrm{UPRT}$ the IC50 is $264 \mu \mathrm{M}$ (Figure 6A), both of which are consistent with data in the literature [10,58]. Mutant EMS7.5 has an IC50 of $217 \mu \mathrm{M}$. Interestingly, mutant EMS10 has an IC50 of $61 \mu \mathrm{M}$, which is several fold less than the $\triangle U P R T$ strain.

When we analyzed the SNVs in the genomes of these mutants we indeed found a mutation in the UPRT coding sequence in the genome of mutant EMS7.5, which resulted in an amino acid change at position 98 from Gly into Glu (Figure 6B). The conserved nature of this glycine and high level of FUDR resistance in this mutant supports the conclusion that this is a loss of function mutation. Unexpectedly, for mutant EMS10 we did not identify a mutation in the UPRT gene, which may explain the intermediate IC50 of this clone (see Additional file 2: Table S2 for complete list of SNVs in EMS10). While complete mapping of the nature FUDR resistance in this mutant is beyond the scope of this study, these findings highlight the power of chemical mutagenesis and phenotypic screening to identify both expected and unexpected biological targets.

\section{Genome distribution of mutations}

The data assembled here comprise 1208 naturally occurring SNVs (Figure 3A) and 527 chemically induced mutations (369 in the ENU mutants, Figure 4; 158 in the EMS mutants, Figure 5). Using circos plots we plotted SNVs on the chromosomes together with the gene density [59] (Figure 7). Despite this small sample size we analyzed these plots for clusters of variants into so-called hot- and cold-spots. We reasoned that hot regions could be low in gene coding sequence and vice versa. Based on these data we do not observe any obvious hot or cold spots for either naturally occurring or chemically induced SNVs, and as such we detect no clear correlation between SNV location and gene density. While we did not observe signatures of bias at any specific regions of the genome, we sought to further test the randomness of
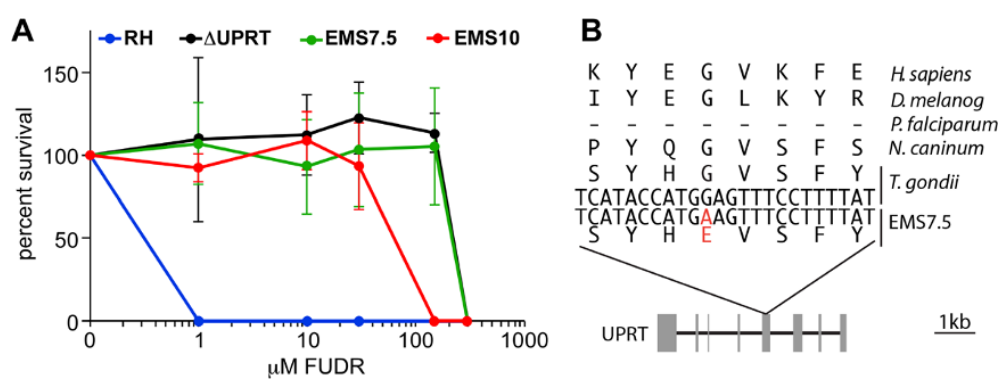

Figure 6 FUDR resistant mutants. (A) The resistance of Toxoplasma strains to varying concentrations of FUDR. Survival was determined by plaque formation relative to an untreated control. Error bars represent SD from 6 independent experiments. Both strains were initially selected for survival in the presence of $20 \mu \mathrm{M}$ FUDR. (B) Proposed etiological mutation in UPRT identified in mutant EMS7.5. Genbank accession numbers for HsUPRT and DmUPRT are NP_659489 and CG5537, respectively. 


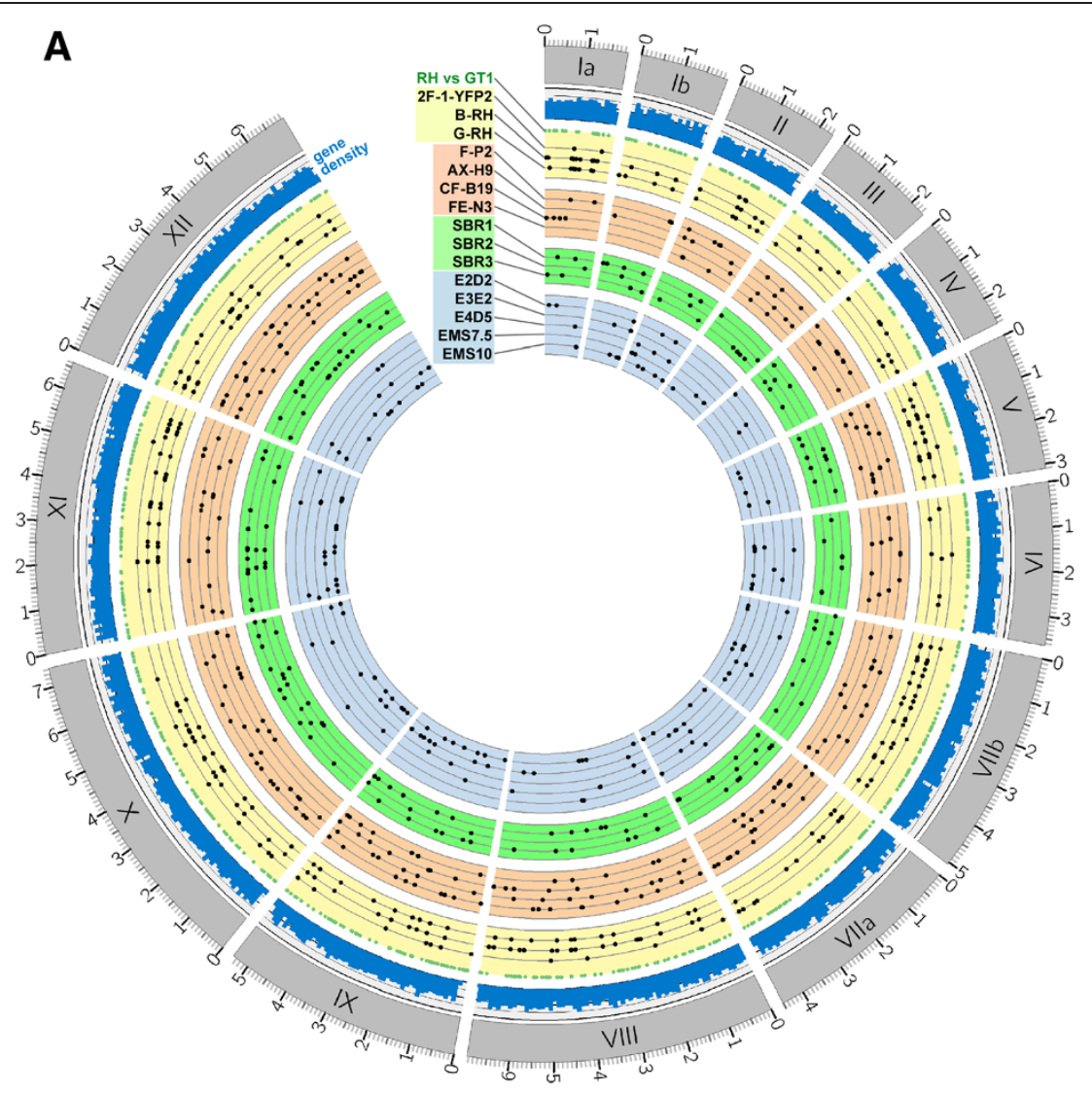

B

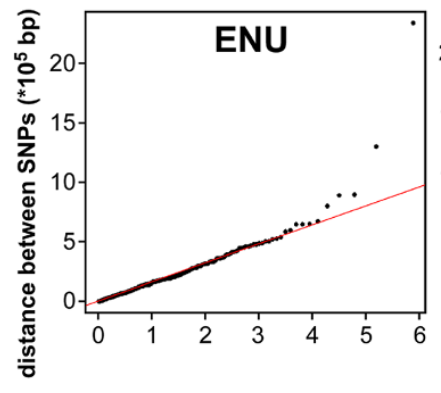

C

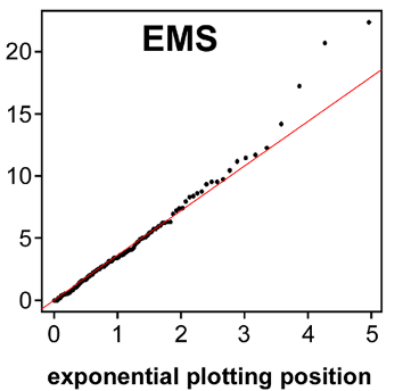

D

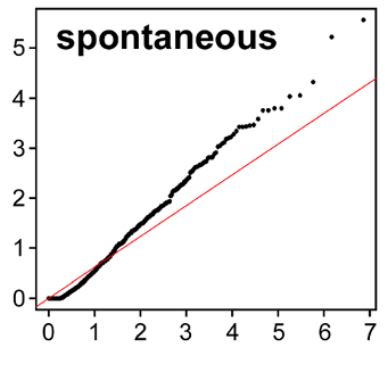

Figure 7 Genome wide distribution of mutations. (A) Circos plot of all mutants and gene density. The Toxoplasma GT1 reference genome is composed of the 14 chromosomes displayed in the outermost circle. The SNVs shared between all 3 RH parent lines and the GT1 reference are represented by green dots. Strain and mutant specific variations are represented by black dots and are grouped as either parent lines (yellow) or by mutant genealogy and mutagen as shown in Figure 1. Gene density was mapped using a sliding window approach with a window size of 100 kb. (B-D) QQ-plot of SNV distances vs. exponential distribution. To test the assumption that mutations are distributed randomly, the inter-SNV distances (taken from Additional file 2: Table S2) are plotted against the exponential distribution, with $\lambda=1 /$ mean, using a Quantile-Quantile plot; $\mathrm{B}=$ distances between ENU induced SNVs $(\mathrm{N}=368$, mean $=159582), \mathrm{C}=$ distances between EMS induced SNVs $(N=144$, mean $=359992.3)$, $\mathrm{D}=$ distances between shared SNVs between the parent strains $(N=968$, mean $=61631.86)$. The solid red line in each plot represents the null assumption $y=x$.

the distribution of mutations across the genome as a whole. Randomly distributed mutations follow a Poisson distribution with $\lambda$ equal to the SNV rate. It thus follows that the distance between randomly distributed SNVs will be exponentially distributed with a rate equal to $1 / \lambda[60]$. Quantile-Quantile (QQ) plots of the inter-mutational distances for each sample versus the exponential distribution are plotted in Figure 7B-D. The mutations in the parent samples accumulated during normal in vitro culture and have been subject to the selective pressures associated with that environment for numerous generations. As expected these variants do not appear randomly distributed, demonstrated by their divergence from the exponential distribution (Figure 7D). This indicates that these variants 
are not randomly distributed. Both the ENU- and EMSderived SNV distributions closely match the theoretical random distribution (Figure $7 \mathrm{~B}, 7 \mathrm{C}$ ), indicating that the induced random mutations are, indeed, randomly distributed. Using the two-sample Kolmogorov-Smirnov test for each sample against the assumption of an exponential distribution, ENU has p-value $=0.7107$ and EMS has $\mathrm{p}$-value $=0.8782$ indicating that they do follow the exponential distribution and are thus randomly distributed. The SNVs shared between the parent samples have a $\mathrm{p}$-value $=2.2 \mathrm{e}-16$ indicating that these mutations are not exponentially distributed and thus not random. To assure ourselves that the difference in distribution between the mutagen-derived SNVs and the in vitro culture derived SNVs is not due to sample size, we repeatedly downsampled the in vitro set to match the number of SNVs in the two mutagenesis data sets and this did not affect the conclusions. Overall, these data show that ENU and EMS induce a random distribution of mutations across all chromosomes, whereas the accumulation of SNVs over time in cell culture is non-random, consistent with selection.

\section{Discussion}

The goal of this study was to better understand the nature of chemical mutagenesis to make informed decisions in the fine-tuning of forward genetic studies in Toxoplasma. Additionally we characterized several other salient effects of laboratory manipulation on the Toxoplasma genome. We identified significant variation between the supposedly identical lab strains passaged in different labs. Relatively small numbers of completely unique mutations (19-66) were observed when analyzing individual parent strains. However, B-RH and G-RH, which are derived from the same clone, share an additional $85 \mathrm{SNVs}$ not found in the 2F-1-YFP2 strain. These 85 mutations originated before this line was distributed to different labs. These lab-strains were generated and separated 10-15 years ago, which encompasses a variable and unknown number of generations, preventing us from making an accurate estimate of the mutation rate per generation.

We identified almost 1,000 variations among three different versions of the lab adapted RH strain as compared to the reference strain GT1 (Figure 1). This value closely resembles the number of mutations based on a more limited data set [61] and our data further validates this number. The variations observed in the parent strains were non-randomly distributed across the genome, with a bias away from coding regions (Figures 3,7 ). This is consistent with the principle of purifying selection, wherein amino acid changes confer a competitive disadvantage [62]. Our data shows a genome-wide ti/tv ratio close to 1 for spontaneous mutations. No previous reports for ti/tv ratios in Toxoplasma are available, but this ratio is within the normal range of ratios seen in other organisms [53,54].
We attempted to determine whether the selection of parasites with different drugs might influence mutagenic profiles (Figure 1). For instance, 6-TX and FUDR are nucleotide analogs that could result in mutations in the genome, whereas phleomycin is a validated mutagen in Toxoplasma, causing double-strand breaks [63]. We were unable to detect any definitive mutagenic signatures for any of these drug selections, though a slight decrease in $\mathrm{A} / \mathrm{T}$ mutation preference was observed in FUDR selected mutants. While the $\mathrm{A} / \mathrm{T} \%$ and ti/tv ratio for $2 \mathrm{~F}-1-\mathrm{YFP} 2$ differed from the other lab strains, it is unlikely that this is due to its selection with phleomycin. Single nucleotide mutations are not an expected consequence of double strand break repair. In our initial analysis we did screen for short indels, however none of the calls reached high confidence values and none could be experimentally validated, likely due to the significant divergence between the $\mathrm{RH}$ strain and the GT1 reference. Attempts to include indels called by the current software package increased our false positive rate without contributing any valid calls, and thus indel calling was not used in this analysis. New software packages, which directly compare mutant and parent rather than aligning both to the reference such as NIKS [64] or RUFUS (Farrell, Marth et al., in preparation), will most likely perform better for indels if the reference is of low quality.

Interestingly, we detected 50 polymorphisms between the apicoplast genome of GT1 and the RH strain. Since the apicoplast genome is contained on $35 \mathrm{~kb}$ of circular DNA [65] this indicates a 100-fold higher spontaneous mutagenesis rate in the plastid's genome than in the nuclear genome. This may highlight differences in the fidelity of plastid genome replication or to a unique deployment of DNA repair enzymes in this organelle. Another possible explanation for the high rate of mutations observed in the apicoplast is the polyploidy of the plastid genome. The relative number of sequence reads mapping to the plastid suggest 7, 14, and 20 copies of its genome in B-RH, G-RH, and n-F-P2, respectively. Ploidy of up to $25 \mathrm{~N}$ is consistent with previous reports [66,67], although the variation is quite wide across strains.

Our finding that $\sim 74 \%$ of the EMS mutations are at an A/T base pair in Toxoplasma (Figure 5) deviates dramatically from the general observation across other systems where EMS has a GC-biased signature [21-23]. The mechanism of EMS mutagenic activity is the reaction of its ethyl group predominantly with guanine and to a much lower extent with adenine to generate $\mathrm{N}$-alkyl adducts [68]. During DNA replication, DNA polymerases frequently place thymine, instead of cytosine, opposite ethylguanine resulting in transitions. The presence of 3 -alkyl-adenine is not immediately mutagenic in mammalian cells [69]. It has been suggested that this could be due to an SOS-like response turned on by cytotoxic 
lesions like 3-alkyladenine, or, alternatively, that increased removal of 3-alkyladenine increases the number of single-strand breaks in DNA, which stalls DNA replication and allows a prolonged time for DNA repair by the alkyltransferase [69]. It is currently neither known how Toxoplasma DNA polymerase handles different kinds of DNA damage, nor whether Toxoplasma mounts an SOS response in response to DNA damage. Toxoplasma possesses two apurinic/apyrimidinic (AP) endonucleases that function in DNA base excision repair: exonuclease III (TgAPE) and endonuclease IV (TgAPN) [70] but their activities against ENU or EMS induced damage have not been determined. Collectively, these findings indicate that the distinct EMS mutagenic signature in Toxoplasma is most likely caused by a difference in DNA repair pathways and suggest that Toxoplasma is able to repair G-alkylation quite well and A-alkylation very poorly. The signature of mutagenesis by both EMS and ENU clearly differs from the accumulation of SNVs through normal in vitro culture, where the $\mathrm{A} / \mathrm{T}$ bias nearly mirrors the $45 \% \mathrm{~A} / \mathrm{T}$ composition of the genome as a whole.

One of the new insights generated here is the relationship between particular dosages of ENU or EMS (as measured by percent killing) and the total number of resultant mutations. In the past, estimates of this number have been made experimentally using pro-drugs like 6-TX and FUDR targeting the negative selectable markers HXGPRT and UPRT, respectively $[8,11,15]$. This led to an estimated 10-100 mutations per genome (65 Mb; haploid) using an ENU dosage inducing $70 \%$ killing, but since it was unknown which mutations resulted in drug resistance these estimates were very rough. The data shown here confirm that these estimates were accurate, as we always observe less than 100 SNVs per genome at the highest dosage used (70\% ENU killing and 90\% EMS killing). This approximates 1 mutation per $\mathrm{Mb}$, which appears to be the "magic number" as it is consistently observed in mutagenesis studies that aim to generate phenotypic mutants across organisms. This includes ENU mutagenized mice (roughly 1 mutation per $\mathrm{Mb}$ [71]), a combination of mutagens in the yeast Pichia stipites (14 mutations in the $15 \mathrm{Mb}$ genome [25]), and EMS mutagenesis of the yeast Saccharomyces cerevisiae (1 mutation per $\mathrm{Mb}$ [72]).

We also generated FUDR resistant mutants in this study as an internal control for mutagenesis experiments at high EMS dosages. Mutants EMS7.5 and EMS10 were treated with $20 \mu \mathrm{M}$ FUDR to select for mutations in the UPRT gene (Figures 5, 6). The Gly98 into Glu UPRT mutation in EMS7.5 is consistent with resistance to FUDR since this residue lies in $\beta$-arm of the dimerization interface, and the crystal structure indicates that dimerization is required for substrate binding and enzymatic activity [73]. However, in mutant EMS10 we did not identify a mutation in UPRT.
This mutant is also less resistant to FUDR than the complete UPRT gene deletion or mutant EMS7.5 (Figure 6), hinting at a potentially different resistance mechanism. The list of non-synonymous variations contains many hypothetical genes, which could potentially be involved in FUDR resistance, but the list also contains CPSII, which is the first enzyme in the de novo pyrimidine synthesis pathway (Lys1327 into Arg). It is known that the cytotoxicity of FUDR and 5-FU can be pluriform and the details are poorly understood [74]. Whether the mutation in CPSII indeed confers FUDR resistance is outside the scope of the current study and was not further pursued here.

Our data show that chemical mutagenesis with either ENU or EMS induces mutations randomly distributed over the genome (Figure 7). From a practical forward genetic perspective that is the desired situation. Because of its use in Pfefferkorn's early experiments, ENU became the mutagen of choice in Toxoplasma [5]. In this study we confirm Pfefferkorn's later findings that efficient mutagenesis was also obtained with EMS treatment of actively growing intracellular parasites ([15]; nitrosoguanidine treatment of extracellular parasites was on par with EMS). We also show that, compared to ENU, EMS produces a similar or slightly higher fraction of non-synonymous mutations, which is preferred in chemical mutagenesis experiments for mutant screens. From a practical perspective, the ideal number of SNVs for genetic studies in Toxoplasma is $5-10$ non-synonymous mutations per genome $[7,13,33]$. To be within this range, the optimal dosage of EMS based on our data would be an EMS concentration inducing $80 \%$ killing (Figure 5). However, we would like to note this recommendation is based on only two data points at $80 \%$ and $90 \%$ killing causing 9 and 17 non-synonymous mutations, respectively. If for other experimental considerations ENU should be the preferred mutagen, we recommend an ENU dosage inducing 60\% killing to be within the 5-10 non-synonymous mutation range.

\section{Conclusions}

We successfully applied whole genome sequencing and mutational profiling methods to Toxoplasma and identified genome variations acquired during long-term in vitro culture and upon chemical mutagenesis. Comparison of the three different lab strains showed that serial passaging of parasites over 10-15 years has resulted in the accumulation of 50-100 SNVs, predominantly in non-coding sequence or as silent mutations with respect to amino acid code. This is the first time we are able to provide insights on the accumulation of polymorphisms in cultured strains of $T$. gondii, which are important variables that could underlie potential differences in experimental outcome in different labs. Although the experiments here were not explicitly designed to detect mutagenic profiles for different drug selectable markers, a subtle change in A/T bias was 
detected following FUDR selection in EMS-generated mutants. No such signatures were identified for 6-TX or phleomycin selection, suggesting any mutagenic effects of drug selection are minimal in comparison to those generated by the underlying mutagen.

As in other organisms, the ENU mutagenic profile in Toxoplasma showed a consistent A/T bias. Interestingly, a similar A/T bias was observed with EMS, which represents a stark departure from the neutral or G/C bias commonly observed in other systems. This suggests the DNA repair of EMS damage in Toxoplasma differs from other well-studied genetic systems. Of importance for the design and interpretation of forward genetic experiments, we validated that both ENU and EMS induce mutations randomly over the genome. For EMS we observed a slightly higher rate of non-synonymous point mutations and a lower rate of mutation in non-coding sequence compared to ENU. These differences, while subtle, make EMS more likely to generate coding changes while minimizing noncoding SNVs that can be challenging to functionally dissect. EMS is therefore a preferable mutagen for forward genetic experiments where non-synonymous mutations are the desired outcome. From our experience, an $80 \%$ killing dose of EMS, which yields approximately $35 \mathrm{SNVs}$, will cause 5-10 coding changes. Following phenotypic selection, this is a feasible number of genes upon which to focus. We believe that the insights presented here will not only be helpful to studies in the relatively well-accessible Toxoplasma parasite, but will pave the way toward forward genetic studies in less accessible protozoan pathogens as well.

\section{Availability of supporting data}

Sequence reads have been deposited at the National Center for Biotechnology Information sequence read archive under accession numbers SRR346134 (2F-1-YFP2), SRR346136 (o-F-P2), SUB465503 (n-F-P2), SUB46416 (B-RH), SUB46 4162 (G-RH) and SAMN02647169 - SAMN02647179 for all other strains.

\section{Additional files}

Additional file 1: Table S1. SNV validation materials and results for mutants E2D2, E3E2, and E4D5. Given are the chromosomal localization of the SNV call, primer sequences used for PCR amplification of predicted SNV from mutant and parent line, and whether the SNV validated by Sanger sequencing of the PCR products.

Additional file 2: Table S2. SNV calling and annotation data for all mutants and parent lines. Includes all data presented in Figures 3, 4, 5, and 7. Depths reported are the total read coverage at the given location for Parent and Mutant sample in each experiment respectively. Prob is the phred scaled probability of the variation as reported by FREEBAYES.

\section{Abbreviations}

6-TX: 6-ThioXanthine; bp: base pair; Chl: Chloramphenicol; ENU: N-ethyl-Nnitrosourea; EMS: Ethylmethane sulfonate; FUDR: 5-fluoro-2'-deoxyuridine;
HFF: Human foreskin fibroblast; HXGPRT: Hypoxanthine-xanthine-guanine phosphoribosyltransferase; Phle: Phleomycin; SNV: Single nucleotide variation; UPRT: Uracil phosphoribosyl transferase.

\section{Competing interests}

The authors declare that they have no competing interests.

\section{Author's contributions}

MJG generated all previously unreported mutants except the SBR mutants, which were generated and analyzed by KB and IJB. AF and GM performed the sequence data analysis and SNV calling. BB and BIC validated the SNV calls by PCR and Sanger sequencing; BIC performed FUDR IC50 assays. AF, $\mathrm{BIC}$, and MJG wrote the manuscript. All authors provided comments on the paper, read, and approved the final manuscript.

\section{Acknowledgements}

We thank Drs. Patrick Gaffney and Graham Wiley of the "Oklahoma Medical Research Foundation", and Drs. Chad Nussbaum and Carsten Russ of the "Broad Sequencing Platform" for sequencing. This work was supported by National Institutes of Health grants Al081220 to MJG and GTM, Al099658 to MJG, HG004719 to GTM, Al069986 to IJB and an American Heart Association Scientist Development Grant (0635480 N) and an American Cancer Society Research Scholar Grant (RSG-12-175-01 - MPC) to MJG.

\section{Author details}

'Department of Biology, Boston College, Higgins Hall 355, 140

Commonwealth Avenue, Chestnut Hill, MA 02467, USA. ${ }^{2}$ Department of Microbiology and Immunology, University of Oklahoma Health Sciences Center, 940 Stanton L. Young Blvd., BMSB 1053, Oklahoma City, OK 73104, USA. ${ }^{3}$ Present address: Department of Molecular Microbiology, Washington University School of Medicine, MPRB 9230, 4940 Parkview Place, St. Louis, MO 63110, USA. ${ }^{4}$ Present address: Department of Microbiology and Immunology, State University of New York, 138 Farber Hall, 3435 Main Street, Buffalo, NY 14214, USA.

Received: 23 December 2013 Accepted: 2 May 2014

Published: 10 May 2014

\section{References}

1. White NJ, Pukrittayakamee S, Hien TT, Faiz MA, Mokuolu OA, Dondorp AM: Malaria. Lancet 2013, 383:723-735.

2. Montoya JG, Liesenfeld O: Toxoplasmosis. Lancet 2004, 363:1965-1976.

3. Kim K, Weiss LM: Toxoplasma gondii: the model apicomplexan. Int J Parasitol 2004, 34:423-432

4. Pfefferkorn ER, Pfefferkorn LC: Arabinosyl nucleosides inhibit Toxoplasma gondii and allow the selection of resistant mutants. J Parasitol 1976, 62:993-999

5. Pfefferkorn ER, Pfefferkorn LC: Toxoplasma gondii: isolation and preliminary characterization of temperature-sensitive mutants. Exp Parasitol 1976, 39:365-376.

6. Pfefferkorn ER, Schwartzman JD, Kasper LH: Toxoplasma gondii: use of mutants to study the host-parasite relationship. Ciba Found Symp 1983, 99:74-91.

7. Farrell A, Thirugnanam S, Lorestani A, Dvorin JD, Eidell KP, Ferguson DJ, Anderson-White BR, Duraisingh MT, Marth GT, Gubbels MJ: A DOC2 protein identified by mutational profiling is essential for apicomplexan parasite exocytosis. Science 2012, 335:218-221.

8. Gubbels MJ, Lehmann M, Muthalagi M, Jerome ME, Brooks CF, Szatanek T, Flynn J, Parrot B, Radke J, Striepen B, White MW: Forward genetic analysis of the apicomplexan cell division cycle in Toxoplasma gondii. PLoS Pathog 2008, 4:e36.

9. White MW, Jerome ME, Vaishnava S, Guerini M, Behnke M, Striepen B: Genetic rescue of a Toxoplasma gondii conditional cell cycle mutant. Mol Microbiol 2005, 55:1060-1071.

10. Pfefferkorn ER, Pfefferkorn LC: Toxoplasma gondii: characterization of a mutant resistant to 5-fluorodeoxyuridine. Exp Parasitol 1977, 42:44-55.

11. Black MW, Arrizabalaga G, Boothroyd JC: lonophore-resistant mutants of Toxoplasma gondii reveal host cell permeabilization as an early event in egress. Mol Cell Biol 2000, 20:9399-9408. 
12. Arrizabalaga G, Ruiz F, Moreno S, Boothroyd JC: lonophore-resistant mutant of Toxoplasma gondii reveals involvement of a sodium/ hydrogen exchanger in calcium regulation. J Cell Biol 2004, 165:653-662.

13. Garrison E, Treeck M, Ehret E, Butz H, Garbuz T, Oswald BP, Settles M, Boothroyd J, Arrizabalaga G: A forward genetic screen reveals that calcium-dependent protein kinase 3 regulates egress in Toxoplasma. PLoS Pathog 2012, 8:e1003049.

14. Uyetake L, Ortega-Barria E, Boothroyd JC: Isolation and characterization of a cold-sensitive attachment/invasion mutant of Toxoplasma gondii. Exp Parasitol 2001, 97:55-59.

15. Pfefferkorn ER, Pfefferkorn LC: Quantitative studies of the mutagenesis of Toxoplasma gondii. J Parasitol 1979, 65:364-370.

16. Sikora P, Chawade A, Larsson M, Olsson J, Olsson O: Mutagenesis as a Tool in Plant Genetics, Functional Genomics, and Breeding. Int J Plant Genomics 2011, 13

17. Anderson P: Mutagenesis. Methods Cell Biol 1995, 48:31-58

18. Flibotte S, Edgley ML, Chaudhry I, Taylor J, Neil SE, Rogula A, Zapf R, Hirst M, Butterfield Y, Jones SJ, Marra MA, Barstead RJ, Moerman DG: Wholegenome profiling of mutagenesis in Caenorhabditis elegans. Genetics 2010, 185:431-441.

19. Acevedo-Arozena A, Wells S, Potter P, Kelly M, Cox RD, Brown SD: ENU mutagenesis, a way forward to understand gene function. Annu Rev Genomics Hum Genet 2008, 9:49-69.

20. Amsterdam A, Hopkins N: Mutagenesis strategies in zebrafish for identifying genes involved in development and disease. Trends Genet 2006, 22:473-478.

21. Barbaric I, Wells S, Russ A, Dear TN: Spectrum of ENU-induced mutations in phenotype-driven and gene-driven screens in the mouse. Environ $\mathrm{Mol}$ Mutagen 2007, 48:124-142.

22. Greene EA, Codomo CA, Taylor NE, Henikoff JG, Till BJ, Reynolds SH, Enns LC, Burtner C, Johnson JE, Odden AR, Comai L, Henikoff S: Spectrum of chemically induced mutations from a large-scale reverse-genetic screen in Arabidopsis. Genetics 2003, 164:731-740.

23. Hanash SM, Boehnke M, Chu EH, Neel JV, Kuick RD: Nonrandom distribution of structural mutants in ethylnitrosourea-treated cultured human lymphoblastoid cells. Proc Natl Acad Sci U S A 1988, 85:165-169.

24. Hillier LW, Marth GT, Quinlan AR, Dooling D, Fewell G, Barnett D, Fox P, Glasscock JI, Hickenbotham M, Huang W, Magrini VJ, Richt RJ, Sander SN, Stewart DA, Stromberg M, Tsung EF, Wylie T, Schedl T, Wilson RK, Mardis ER: Whole-genome sequencing and variant discovery in C. elegans. Nat Methods 2008, 5:183-188

25. Smith DR, Quinlan AR, Peckham HE, Makowsky K, Tao W, Woolf B, Shen L, Donahue WF, Tusneem N, Stromberg MP, Stewart DA, Zhang L, Ranade SS, Warner JB, Lee CC, Coleman BE, Zhang Z, McLaughlin SF, Malek JA, Sorenson JM, Blanchard AP, Chapman J, Hillman D, Chen F, Rokhsar DS, McKernan KJ, Jeffries TW, Marth GT, Richardson PM: Rapid whole-genome mutational profiling using next-generation sequencing technologies. Genome Res 2008, 18:1638-1642.

26. Sarin S, Prabhu S, O'Meara MM, Pe'er I, Hobert O: Caenorhabditis elegans mutant allele identification by whole-genome sequencing. Nat Methods 2008, 5:865-867

27. Shen Y, Sarin S, Liu Y, Hobert O, Pe'er I: Comparing platforms for C. elegans mutant identification using high-throughput whole-genome sequencing. PLoS One 2008, 3:e4012.

28. Sarin S, Bertrand V, Bigelow H, Boyanov A, Doitsidou M, Poole RJ, Narula S, Hobert $O$ : Analysis of multiple ethyl methanesulfonate-mutagenized Caenorhabditis elegans strains by whole-genome sequencing. Genetics 2010, 185:417-430.

29. Zuryn S, Le Gras S, Jamet K, Jarriault S: A strategy for direct mapping and identification of mutations by whole-genome sequencing. Genetics 2010 186:427-430.

30. Voz ML, Coppieters W, Manfroid I, Baudhuin A, Von Berg V, Charlier C, Meyer D, Driever W, Martial JA, Peers B: Fast homozygosity mapping and identification of a zebrafish ENU-induced mutation by whole-genome sequencing. PLoS One 2012, 7:e34671.

31. Shiwa Y, Fukushima-Tanaka S, Kasahara K, Horiuchi T, Yoshikawa $H$ : Whole-Genome Profiling of a Novel Mutagenesis Technique Using Proofreading-Deficient DNA Polymerase delta. Int J Evol Biol 2012, 2012:860797.

32. Hobert $\mathrm{O}$ : The impact of whole genome sequencing on model system genetics: get ready for the ride. Genetics 2010, 184:317-319.
33. Brown KM, Suvorova ES, Farrell A, Wiley GB, Gubbels MJ, Marth GT, Gaffney PM, White M, Blader IJ: Chemical genetic screening identifies a small molecule that blocks Toxoplasma growth by inhibiting both host- and parasite-encoded kinases. PLoS Path 2014, accepted for publication.

34. Sugi T, Kobayashi K, Takamea H, Gong H, Ishiwa A: Identification of mutations in TgMAPK1 of Toxoplasma gondii conferring the resistance to 1NM-PP1. Int J Parasitol Drugs Drug Resist 2013, 3:93-101.

35. Coleman BI, Gubbels MJ: A genetic screen to isolate Toxoplasma gondii host cell egress mutants. J Vis Exp 2012, 60:e3807

36. Roos DS, Donald RG, Morrissette NS, Moulton AL: Molecular tools for genetic dissection of the protozoan parasite Toxoplasma gondii. Methods Cell Biol 1994, 45:27-63.

37. Sabin AB: Toxoplasmic encephalitis in children. J Am Med Assoc 1941, 116:801-807.

38. Donald RG, Carter D, Ullman B, Roos DS: Insertional tagging, cloning, and expression of the Toxoplasma gondii hypoxanthine-xanthine-guanine phosphoribosyltransferase gene. Use as a selectable marker for stable transformation. J Biol Chem 1996, 271:14010-14019.

39. Dobrowolski JM, Sibley LD: Toxoplasma invasion of mammalian cells is powered by the actin cytoskeleton of the parasite. Cell 1996, 84:933-939.

40. Teo CF, Zhou XW, Bogyo M, Carruthers VB: Cysteine protease inhibitors block Toxoplasma gondii microneme secretion and cell invasion. Antimicrob Agents Chemother 2007, 51:679-688.

41. Gubbels MJ, Li C, Striepen B: High-throughput growth assay for Toxoplasma gondii using yellow fluorescent protein. Antimicrob Agents Chemother 2003, 47:309-316.

42. Eidell KP, Burke T, Gubbels MJ: Development of a screen to dissect Toxoplasma gondii egress. Mol Biochem Parasitol 2010, 171:97-103.

43. Carey KL, Westwood NJ, Mitchison TJ, Ward GE: A small-molecule approach to studying invasive mechanisms of Toxoplasma gondii. Proc Natl Acad Sci U S A 2004, 101:7433-7438.

44. Lee W-P, Stromberg M, Ward A, Stewart C, Garrison E, Marth GT: MOSAIK: A hash-based algorithm for accurate next-generation sequencing shortread mapping. PLoS One 2014, 9:e90581.

45. Marth GT, Korf I, Yandell MD, Yeh RT, Gu Z, Zakeri H, Stitziel NO, Hillier L, Kwok PY, Gish WR: A general approach to single-nucleotide polymorphism discovery. Nat Genet 1999, 23:452-456.

46. Garrison E, Marth GT: Haplotype-Based Variant Detection from Short-Read Sequencing. 2012. http://arxiv.org/abs/1207.3907v2.

47. Krzywinski M, Schein J, Birol I, Connors J, Gascoyne R, Horsman D, Jones SJ, Marra MA: Circos: an information aesthetic for comparative genomics. Genome Res 2009, 19:1639-1645.

48. Gajria B, Bahl A, Brestelli J, Dommer J, Fischer S, Gao X, Heiges M, lodice J, Kissinger JC, Mackey AJ, Pinney DF, Roos DS, Stoeckert CJ Jr, Wang H, Brunk BP: ToxoDB: an integrated Toxoplasma gondii database resource. Nucleic Acids Res 2008, 36:D553-D556.

49. Sibley LD, Boothroyd JC: Virulent strains of Toxoplasma gondii comprise a single clonal lineage. Nature 1992, 359:82-85.

50. Martinelli $A$, Henriques $G$, Cravo $P$, Hunt $P$ : Whole genome re-sequencing identifies a mutation in an $\mathrm{ABC}$ transporter (mdr2) in a Plasmodium chabaudi clone with altered susceptibility to antifolate drugs. Int J Parasitol 2010, 41:165-171.

51. Araya CL, Payen C, Dunham MJ, Fields S: Whole-genome sequencing of a laboratory-evolved yeast strain. BMC Genomics 2010, 11:88.

52. Le Crom S, Schackwitz W, Pennacchio L, Magnuson JK, Culley DE, Collett JR, Martin J, Druzhinina IS, Mathis H, Monot F, Seiboth B, Cherry B, Rey M, Berka $R$, Kubicek CP, Baker SE, Margeot A: Tracking the roots of cellulase hyperproduction by the fungus Trichoderma reesei using massively parallel DNA sequencing. Proc Natl Acad Sci U S A 2009, 106:16151-16156.

53. Yang Z, Yoder AD: Estimation of the transition/transversion rate bias and species sampling. J Mol Evol 1999, 48:274-283.

54. Zhang J: Rates of conservative and radical nonsynonymous nucleotide substitutions in mammalian nuclear genes. J Mol Evol 2000, 50:56-68.

55. Khan A, Taylor S, Su C, Sibley LD, Paulsen I, Ajioka JW: Genetics and Genome Organization of Toxoplasma Gondii. Norwich, UK: Horizon Scientific Press; 2007.

56. Pfefferkorn ER: Toxoplasma gondii: the enzymic defect of a mutant resistant to 5-fluorodeoxyuridine. Exp Parasitol 1978, 44:26-35.

57. Schwartzman JD, Pfefferkorn ER: Pyrimidine synthesis by intracellular Toxoplasma gondii. J Parasitol 1981, 67:150-158. 
58. Donald RG, Roos DS: Insertional mutagenesis and marker rescue in a protozoan parasite: cloning of the uracil phosphoribosyltransferase locus from Toxoplasma gondii. Proc Natl Acad Sci U S A 1995, 92:5749-5753.

59. Goecks J, Eberhard C, Too T, Nekrutenko A, Taylor J: Web-based visual analysis for high-throughput genomics. BMC Genomics 2013, 14:397.

60. Sun YV, Levin AM, Boerwinkle E, Robertson H, Kardia SL: A scan statistic for identifying chromosomal patterns of SNP association. Genet Epidemiol 2006, 30:627-635.

61. Yang N, Farrell A, Niedelman W, Melo M, Lu D, Julien L, Marth GT, Gubbels MJ, Saeij JP: Genetic basis for phenotypic differences between different Toxoplasma gondii type I strains. BMC Genomics 2013, 14:467.

62. Martincorena I, Seshasayee AS, Luscombe NM: Evidence of non-random mutation rates suggests an evolutionary risk management strategy. Nature 2012, 485:95-98.

63. Fox BA, Ristuccia JG, Gigley JP, Bzik DJ: Efficient gene replacements in Toxoplasma gondii strains deficient for nonhomologous end-joining. Eukaryot Cell 2009, 8:520-529.

64. Nordstrom KJ, Albani MC, James GV, Gutjahr C, Hartwig B, Turck F, Paszkowski U, Coupland G, Schneeberger K: Mutation identification by direct comparison of whole-genome sequencing data from mutant and wild-type individuals using k-mers. Nat Biotechnol 2013, 31:325-330.

65. Kohler S, Delwiche CF, Denny PW, Tilney LG, Webster P, Wilson RJ, Palmer $J D$, Roos DS: A plastid of probable green algal origin in Apicomplexan parasites. Science 1997, 275:1485-1489.

66. Reiff SB, Vaishnava S, Striepen B: The HU protein is important for apicoplast genome maintenance and inheritance in Toxoplasma gondii. Eukaryot Cell 2012, 11:905-915.

67. Matsuzaki M, Kikuchi T, Kita K, Kojima S, Kuroiwa T: Large amounts of apicoplast nucleoid DNA and its segregation in Toxoplasma gondii. Protoplasma 2001, 218:180-191.

68. Pastink A, Heemskerk E, Nivard MJ, Van Vliet CJ, Vogel EW: Mutational specificity of ethyl methanesulfonate in excision-repair-proficient and -deficient strains of Drosophila melanogaster. Mol Gen Genet 1991, 229:213-218.

69. Klungland A, Laake K, Hoff E, Seeberg E: Spectrum of mutations induced by methyl and ethyl methanesulfonate at the hprt locus of normal and tag expressing Chinese hamster fibroblasts. Carcinogenesis 1995, 16:1281-1285

70. Onyango DO, Naguleswaran A, Delaplane S, Reed A, Kelley MR, Georgiadis MM, Sullivan WJ Jr: Base excision repair apurinic/apyrimidinic endonucleases in apicomplexan parasite Toxoplasma gondii. DNA Repair 2011, 10:466-475.

71. Quwailid MM, Hugill A, Dear N, Vizor L, Wells S, Horner E, Fuller S, Weedon J, McMath H, Woodman P, Edwards D, Campbell D, Rodger S, Carey J, Roberts A, Glenister P, Lalanne Z, Parkinson N, Coghill EL, McKeone R, Cox S, Willan J, Greenfield A, Keays D, Brady S, Spurr N, Gray I, Hunter J, Brown SD, Cox RD: A gene-driven ENU-based approach to generating an allelic series in any gene. Mamm Genome 2004, 15:585-591.

72. Timmermann B, Jarolim S, Russmayer H, Kerick M, Michel S, Kruger A, Bluemlein K, Laun P, Grillari J, Lehrach H, Breitenbach M, Ralser M: A new dominant peroxiredoxin allele identified by whole-genome re-sequencing of random mutagenized yeast causes oxidant-resistance and premature aging. Aging (Albany NY) 2010, 2:475-486.

73. Schumacher MA, Carter D, Scott DM, Roos DS, Ullman B, Brennan RG: Crystal structures of Toxoplasma gondii uracil phosphoribosyltransferase reveal the atomic basis of pyrimidine discrimination and prodrug binding. EMBO J 1998, 17:3219-3232.

74. Longley DB, Harkin DP, Johnston PG: 5-fluorouracil: mechanisms of action and clinical strategies. Nat Rev Cancer 2003, 3:330-338.

doi:10.1186/1471-2164-15-354

Cite this article as: Farrell et al:: Whole genome profiling of spontaneous and chemically induced mutations in Toxoplasma gondii. BMC Genomics 2014 15:354.

\section{Submit your next manuscript to BioMed Central and take full advantage of:}

- Convenient online submission

- Thorough peer review

- No space constraints or color figure charges

- Immediate publication on acceptance

- Inclusion in PubMed, CAS, Scopus and Google Scholar

- Research which is freely available for redistribution 